\title{
Correspondence
}

\section{Treatment of renal failure in neonates}

Sir,

Meeks and Sims describe their experiences of acute renal failure in a neonatal intensive care unit; data were abstracted retrospectively from the hospital records over a four and a half year period; and acute renal failure was defined as anuria of 24 hours' duration or concern by a clinician about poor urine output together with a serum urea concentration exceeding $10 \mathrm{mmol} / \mathrm{l}$ or serum potassium exceeding $7.5 \mathrm{mmol} / \mathrm{l}$. ${ }^{1}$ I would like to comment on some of the points raised in their paper.

Between February and December 1985 I carried out a prospective survey of acute renal failure in 388 consecutive admissions to a regional neonatal intensive care unit. The diagnosis was based on at least one of the following three criteria: a serum creatinine concentration persistently rising over a minimum of two days, oliguria $(<1 \mathrm{ml} / \mathrm{kg} /$ hour) or anuria resistant to volume repletion and present for a minimum of eight hours, hyperkalaemia (confirmed on a repeat unhaemolised sample; potassium concentration $>7.5 \mathrm{mmol} / \mathrm{l}$ ) together with at least one of the following two criteria: otherwise unexplained metabolic acidosis $(\mathrm{pH}<7 \cdot 25$, base deficit $>10 \mathrm{mmol} / \mathrm{l})$ or haematuria.

Twenty four $(6 \cdot 2 \%)$ babies developed renal impairment suggestive of acute intrinsic renal failure; six infants $(25 \%)$ survived, all of whom had been managed conservatively. Eleven babies presented anuric, 10 oliguric, and three were non-oliguric. Overwhelming septicaemia was the commonest underlying associate, occurring in eight infants $(33 \%)$, all of whom were of less than $1500 \mathrm{~g}$ birth weight. Four babies $(17 \%)$ suffered severe perinatal asphyxia; all were over 35 weeks' gestation and outborn.

Thirteen of the 24 infants had a plasma urea concentration within the normal range at the time of diagnosis. I would suggest that measurement of plasma urea is of little value in the newborn as it is influenced by numerous nonrenal factors. Misleading rises in the presence of normal renal function may be seen in catabolic states such as trauma and sepsis and in the presence of sequestered blood; conversely, the higher anabolic state of the healthy newborn suggests that relatively smaller increments in serum urea may reflect renal impairment.

The usefulness of three biochemical indices, in the evaluation of the oliguric neonate, was assessed. A fractional sodium excretion exceeding $2.5 \%, 2$ urine to plasma creatinine ratio of less than 20 , and urinary sodium concentration exceeding $40 \mathrm{mmol} / \mathrm{l},{ }^{3}$ have been described as suggestive of acute intrinsic renal failure. Only $57 \%$ of infants with volume repletion resistant oliguria had a fractional sodium excretion of greater than $2.5 \%$ however, and $43 \%$ a urine to plasma creatinine ratio of less than 20 (poor sensitivity). Although all these infants had a urinary sodium concentration exceeding $40 \mathrm{mmol} / \mathrm{l}$ (high sensi- tivity), the median urinary sodium concentration in infants less than 34 weeks' gestation in the first week of life is around $88 \mathrm{mmol} / \mathrm{l}$ (poor specificity). ${ }^{4}$

Retrospective review can only be regarded as a poor index of significant oliguria or anuria. In addition to the possibility of prerenal oliguria, $7 \%$ of normal infants fail to void during the first 24 hours of life; neonates do not empty their bladders completely on voiding; sick infants may develop retention of urine. Furthermore the occurrence of non-oliguric renal failure in the neonate is a recognised entity.

Meeks and Sims describe 30 neonates with acute renal failure over a four and a half year period. Though they do not state the total number of admissions over this period, this is likely to be a considerable underestimate, despite their conviction to the contrary. Norman and Assadi reported a $6 \%$ incidence of acute renal failure in a series of 314 consecutive admissions to a neonatal intensive care unit ${ }^{5}$; this is a figure similar to ours.

The recognition of acute intrinsic renal failure in the neonate is difficult. Failure to consider the problem in an anticipatory manner, with careful monitoring of urine output and serial measurement of serum creatinine is likely to result in continued underdiagnosis.

\section{References}

1 Meeks ACG, Sims DG. Treatment of acute renal failure in neonates. Arch Dis Child 1988;63:1372-6.

2 Mathew OP, Jones AS, James E, et al. Neonatal renal failure: usefulness of diagnostic indices. Pediatrics 1980;65:57-60.

3 Anand SK. Acute renal failure in the neonate. Pediatr Clin North Am 1982;29:791-800.

${ }^{4}$ Modi N. Development of renal function. Br Med Bull 1988;44: 935-56.

5 Norman M, Assadi F. A prospective study of acute renal failure in the newborn infant. Pediatrics 1979;63:475-9.

$$
\begin{array}{r}
\text { N Modi } \\
\text { Department of Paediatrics } \\
\text { and Neonatal Medicine, } \\
\text { Royal Postgraduate Medical School, } \\
\text { Hammersmith Hospital, } \\
\text { Du Cane Road, London W12 OHS }
\end{array}
$$

Drs Meeks and Sims comment:

We read with interest the letter from Dr Modi and would like to reply to some of her comments. Acute renal failure occurred in less than $2 \%$ of all admissions to the neonatal medical and surgical units during the study period. Although this figure is much lower than that of other reports, ${ }^{1}$ the incidence of acute renal failure depends on the diagnostic criteria used. We assumed oliguria of less than 24 hours' duration to be a feature of hyaline membrane disease rather than acute renal failure. ${ }^{2}$ By taking oliguria present for more than eight hours as 